\title{
Pautas y estilos de crianza presentes en las familias hondureñas en el año 2015
}

\author{
Angelo Antonio Moreno León ${ }^{1}$ \\ Yesmy Desiret Ochoa ${ }^{2}$ \\ Dora Suyapa Díaz Quintero ${ }^{3}$
}

\section{RESUMEN}

Con el objetivo de analizar el fenómeno de la crianza en contexto familiar hondureño, mediante las apreciaciones e interpretaciones de los jefes(as) de familia de ocho localidades del país y de los datos recabados en otras ocho localidades representativas de alta convergencia poblacional en Honduras, se ha realizado esta investigación, a partir de dos ejes metodológicos: uno cuantitativo, en el cual se utilizaron elementos de tipo descriptivo y diversas variables que nos permitieron conocer cómo está conformada la familia hondureña. El otro, de corte cualitativo, a través de dos técnicas: grupos colaborativos y entrevistas semiestructuradas; los dos enfoques permitieron comprender los estilos de crianza adquiridas en el contexto familiar hondureño. Uno de los principales hallazgos, consiste en corroborar que las madres y los padres hondureños, no tienen conciencia de que el concepto "criar" se refiere a un acto eminentemente educativo, vivido dentro de un espacio de afecto hacia los hijos. La palabra "criar" es entendida, más bien, como un modo de asegurar la supervivencia de los miembros de una familia.

Palabras clave: crianza, educación, estilos de crianza, pautas de crianza, prácticas de crianza.

\footnotetext{
${ }^{1}$ Beneficiario de una Beca Sustantiva, investigador principal, profesor del Departamento de Filosofía, Facultad de Humanidades y Artes, UNAH angelomoreno@unah.edu.hn

${ }^{2}$ Beneficiaria de una Beca Sustantiva, investigadora secundaria. Profesora del Departamento de Pedagogía, Facultad de Humanidades y Artes, UNAH: Yesmy_ochoa@yahoo.es

${ }^{3}$ Beneficiario de una Beca Sustantiva, investigadora secundaria.Profesora del Departamento de Pedagogía, Facultad de Humanidades y Artes, UNAH: Diazdora189@gmail.com
} 


\section{ABSTRACT}

With the objective of analyzing the upbringing phenomenon in the Honduran family context, through assessments and interpretations of the heads of families of eight locations of de country and the data collected in other eight representatives localities with high population convergence in Honduras. This research has been carried, based on two methodological axes: a quantitative one, in which elements of a descriptive and variables type were used that allowed us to know how is the honduran family is conformed. The other, qualitative, brings you two techniques: collaborative groups and semi-structured interviews; all two allowed to understand the styles of learning acquired in the Honduran family context.

One of the main findings is to corroborate that Honduran mother and fathers are not aware that the concept of "raising" refers to an eminently educational act lived within a space of affection towards children. The word "raise" is understood, rather, as a way to ensure the survival of the members of a family.

Keywords: Upbringing, education, upbringing styles, upbringin pattern, upbringing practices. 


\section{INTRODUCCIÓN}

El artículo que presentamos a continuación pretende dar cuenta de los resultados más importantes obtenidos en la investigación Pautas y estilos de crianza presentes en las familias hondureñas en el año 2015 , el cual tuvo como propósito fundamental contribuir a la comprensión del fenómeno de la crianza en contexto familiar hondureño, descrito por patrones generalizados en la sociedad hondureña.

Los alcances de la investigación, su título y sus componentes encajan en dos de los ejes prioritarios de investigación de la UNAH: una de ellas, relativa al desarrollo (Prioridad 2. Pobreza e inequidad, sub - apartado 7: grupos vulnerables, pobreza e inequidad); y la otra, a la educación (Prioridad 8. Educación y cultura, sub - apartado 7: Población, identidad y cultura). Otro aspecto sobre la conveniencia del estudio radica en la reciente preocupación del Estado, por impulsar políticas de protección social que promueven la asistencia social, la seguridad social, los 9 servicios de protección dirigidas, en concordancia con los criterios nacionales e internacionales, hacia la atención de la población urbana y rural en condición de pobreza, pobreza extrema, vulnerabilidad, riesgo y exclusión social.

Las implicaciones prácticas de esta investigación constituyen un antecedente teórico para la futura toma de decisiones, puesto que los resultados permitirán comprender el protagonismo de las familias hondureñas 0 , por lo menos, de los responsables de la crianza de las niñas y niños (tíos, abuelos, etc.) en la socialización (crianza, educación). Por último, permitirá valorar hasta dónde la familia cubre o no plenamente su papel socializador. Si se verifica la hipótesis de que los padres hondureños se han eclipsado (a causa de muchos factores) de la labor criadora (educadora) de sus hijos, los resultados permitirán en otro momento, hacer una doble propuesta de Educación formal para la crianza de los hijos, determinando un diseño integrador a los estilos de crianza al contexto familiar, en relación a factores sociales, culturales y demográficos, a partir de la inserción de políticas educativas y jurídicas; e indicando pautas metodológicas flexibles para para la mejora del sistema de crianza en el contexto familiar hondureño, articuladas con el sistema educativo formal, no formal e informal.

En lo que sigue, presentaremos lo que, a nuestra manera de ver, refleja la concepción que las hondureñas y los hondureños tienen sobre el acto de criar y sobre las pautas y estilos de crianza usadas por ellos. Estas apreciaciones, son el resultado de la investigación que ha sido realizada por un equipo de investigación científica confirmado por docentes investigadores que siempre han creído que la educación de los 
padres es vital en la formación de ciudadanos responsables de sí mismos y de los demás.

\section{Sobre los estilos de crianza y los tipos de familia}

La familia juega un papel indispensable en la crianza, es decir, en el proceso de adquisición de conocimientos, de modelos, de maneras de hacer, de pensar, de actuar, de normas, de valores, propios a la sociedad en la cual la persona debe vivir. El filósofo de la Educación Olivier Reboul, (2012:14) hace énfasis sobre la importancia de la crianza, ya que ella es el despertar del humano, la salida de su estado inicial, el horizonte de su estado ideal. La crianza es un proceso espontáneo, en el cual no hay programas, no hay objetivos claros, no hay leyes para ello (Reboul, 2012: 16). Para el filósofo Savater, (1997: 55) la crianza imprime una marca casi vital en los niños, la cual se prolonga y se hace evidente en el carácter y en los buenos hábitos reflejados en las conductas.

Que la crianza no tenga programas, ni planes, ni métodos, es quizás lo que constituye, a nuestra manera de ver, el punto débil de esta educación: muchos padres no tienen consciencia de lo que es la crianza; nadie les ha enseñado a criar. Lo hacen espontáneamente y recordando cómo lo hacían los familiares. Es aquí, justamente, en donde debería abrirse el camino para evitar ese atraso en la educación de las niñas y los niños, creando consciencia, en los futuros padres y madres de su labor importante en la crianza, en la educación.

Los estilos de crianza describen, básicamente, el conjunto de técnicas que utilizan los padres y madres en la educación de los hijos. De acuerdo con la clasificación de las conductas, se han identificado cuatro tipos de estilos, los cuales se agrupan según la tipología de las técnicas. Para este estudio, los cuestionarios fueron analizados bajo la teoría de tipología de estilos de crianza propuestos por Charles E. Schaefer (1959) las variables que se toman en cuenta son: control disciplinario y calidez afectiva.

Al combinar las cuatro dimensiones que se muestran en el esquema, es posible distinguir los tipos de crianza más utilizados que se han definido según las prácticas elegidas por los padres en los procesos de educación de sus hijos. De ahí también es posible identificar si los comportamientos presentes en los padres refieren más 0 menos a alguno de los cuatro tipos de crianza que se plantean como referentes y las posibles consecuencias de cada estilo en los procesos de crianza y educación de los hijos. 


\section{Fig. 1. Tipología de estilos de crianza}

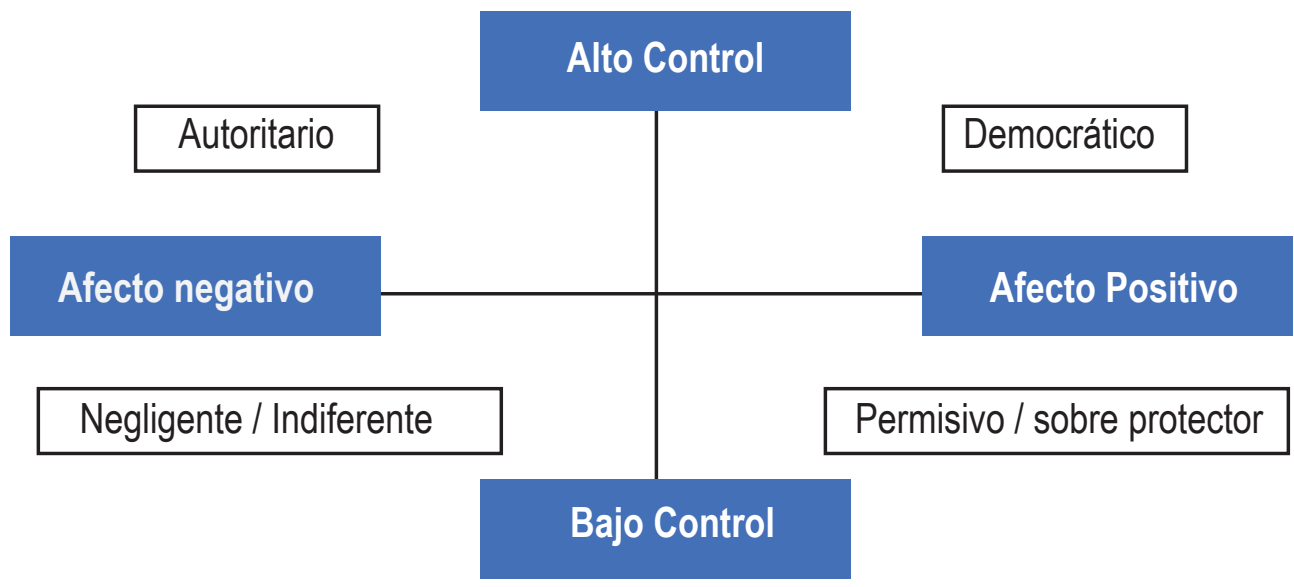

Fuente: elaboración de los investigadores

Por ejemplo, el estilo de crianza democrático presenta un alto nivel de comunicación y afecto. Se refuerzan los comportamientos positivos y se ejerce un control tomando en cuenta los sentimientos y las capacidades de sus hijos. Se evita la arbitrariedad en la toma de decisiones por medio del diálogo. Hay explicación de normas e intercambio de ideas para lograr un consenso.

La crianza autoritaria se caracteriza por presentar altos niveles de control y bajos niveles de afecto y comunicación de los padres con sus hijos. Predomina la exigencia de obediencia y el seguimiento estricto de normas, así como el control y evaluación constante de las actitudes y comportamientos de los hijos. El castigo, así como la aplicación de medidas disciplinarias es importante entre los padres autoritarios y no hay apertura al diálogo.

El estilo de crianza negligente es utilizado por padres que no son receptivos ni exigentes con sus hijos. No demuestran afecto y hay poca comunicación. Los padres suelen ser indiferentes ante la conducta de sus hijos, el apego es escaso y no se establecen normas.

El estilo practicado por padres permisivos se caracteriza por un nivel bajo de control y exigencias de madurez, pero involucra altos niveles de comunicación y afecto con los hijos. Hay poca presencia de castigos, las conductas de los hijos son aceptadas y los padres manifiestan una actitud positiva ante ella. Hay un rechazo del control y ejercicio del poder en la crianza. 


\section{METODOLOGÍA}

Para este estudio, se tomaron en cuenta las apreciaciones e interpretaciones de los jefes (as) de familia de ocho departamentos del país: Comayagua, Copán, Yoro, Cortés, Choluteca, Francisco Morazán, Lempira y Olancho. Se ejecutó la investigación, a partir de dos ejes metodológicos: uno cuantitativo, en el cual se utilizaron elementos de tipo descriptivo y diversas variables que nos permitieron conocer cómo está conformada la familia hondureña. Se aplicaron 1270 instrumentos para obtener información sobre los hábitos de crianza y las prácticas responsivas y de estimulación. Se utilizaron dos tipos de instrumentos: el cuestionario PCRI-M, el cual mide las actitudes de los padres hacia la crianza de los hijos y las dimensiones de control y apoyo. Y el cuestionario sobre Práctica Responsiva y Estimulación (CuPRE), que es un instrumento sobre acciones dirigidas a asegurar la supervivencia y desarrollo infantil y que es considerado un instrumento útil para valorar las prácticas de crianza. El otro, de corte cualitativo, a través de dos técnicas: grupos colaborativos y entrevistas semiestructuradas, aplicadas a padres y madres de las localidades de San Juancito, Fco. Morazán, Sambo Creek, Atlántida, La Ceiba Atlántida, Santa Rita, Yoro, la Esperanza Intibucá y Tegucigalpa, Fco. Morazán. A las tres fases de la primera técnica (identificación, categorización y priorización) y las preguntas generadoras comunes a la técnica de la entrevista semiestructurada, permitieron comprender los estilos de crianza adquiridas en el contexto familiar hondureño. La aplicación de estas técnicas se inició, a partir del planteamiento de las siguientes preguntas generadoras:

- ¿Qué entiende usted por el término criar?

- ¿Qué temas enseña usted en la crianza de sus hijos(as)?

- ¿De esos temas, cuáles cree usted que son decisivos o determinantes para que sus hijos integren a la sociedad?

- ¿Dónde y cómo aprendió usted a criar a sus hijos?

\section{Fase de triangulación de datos en tres etapas y con tres métodos}

El objetivo de esta última etapa fue el de focalizar todos aquellos registros relacionados, por su similitud, con el tema de las pautas y estilos de crianza y agruparlos en un conjunto de datos que tratan, implícita o explícitamente, del mismo tema. En este sentido, cuando se contó con toda la información registrada y con la saturación de datos, se desarrollaron tres momentos claves en el procedimiento de análisis e interpretación de los datos los cuales se pudieron administrar flexiblemente: primer momento: reducción de datos y generación de categorías; segundo momento: 
Figura 2. fases metodológicas del estudio

Proceso en curso: verificación empírica

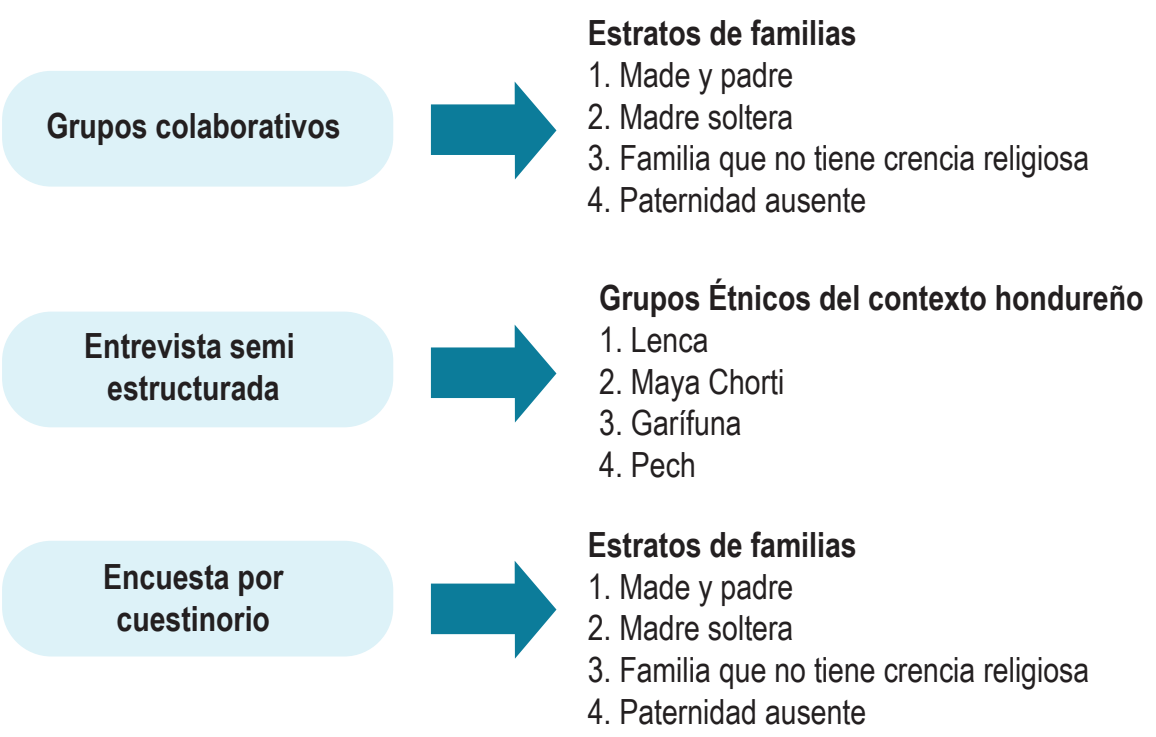

comparación, relación y clasificación de categorías. Surgimiento de las primeras conclusiones; tercer momento: interpretación y discusión de los resultados

La triangulación es la etapa más amplia del proceso de investigación cualitativa, dado que se inicia desde el momento en que se obtienen los primeros datos, hasta consolidar dicho proceso con la obtención de los resultados o conclusiones del estudio

\section{DISCUSIÓN}

Nuestra investigación examinó los hábitos de crianza de los hondureños y hondureñas, según el tipo de familia a la que pertenecen. Para ello, se distinguieron cuatro tipos de familia definidos como: familia nuclear, familia extendida, familia responsable con sus hijos y familia extendida sin padres, identificadas a partir de las características presentes en las personas que participaron en este estudio. La importancia de haber verificado tanto los tipos como los hábitos de crianza que se desarrollan dentro de los distintos tipos de familias establecidos, han permitido determinar si existen similitudes o discrepancias en los tipos de crianza que utilizan los padres y madres 
con sus hijos, según el tipo de familia en el que se desarrollan los procesos de crianza.

Los datos examinados fenomenológicamente nos permiten afirmar que las madres y los padres hondureños no tienen conciencia de que el concepto "criar", se refiere a un acto eminentemente educativo, sino que se entiende más bien como un modo de asegurar la supervivencia de los miembros de una familia y no como un espacio de afecto hacia ellos.

Los valores y actitudes de crianza de las madres y padres hondureños son más de carácter personal que social. Ese dato es revelador, ya que ratifica los altos niveles de individualismo existentes en la sociedad hondureña, alentados por un sistema que los promueven, en detrimento del crecimiento del valor del civismo y la promoción de una ética pública.

En ese sentido, no se encontraron diferencias significativas entre cada uno de los estilos de crianza, según los factores evaluados, teniendo en cuenta el perfil socio económico de los padres y la estructura familiar de los hogares. Eso significa que las pautas autoritarias $(35.4 \%)$ y, en su mayoría, las democráticas (48.4\%), se mantienen, a pesar de esas diferencias.

\section{Grafico 1. Estilos de crianza}

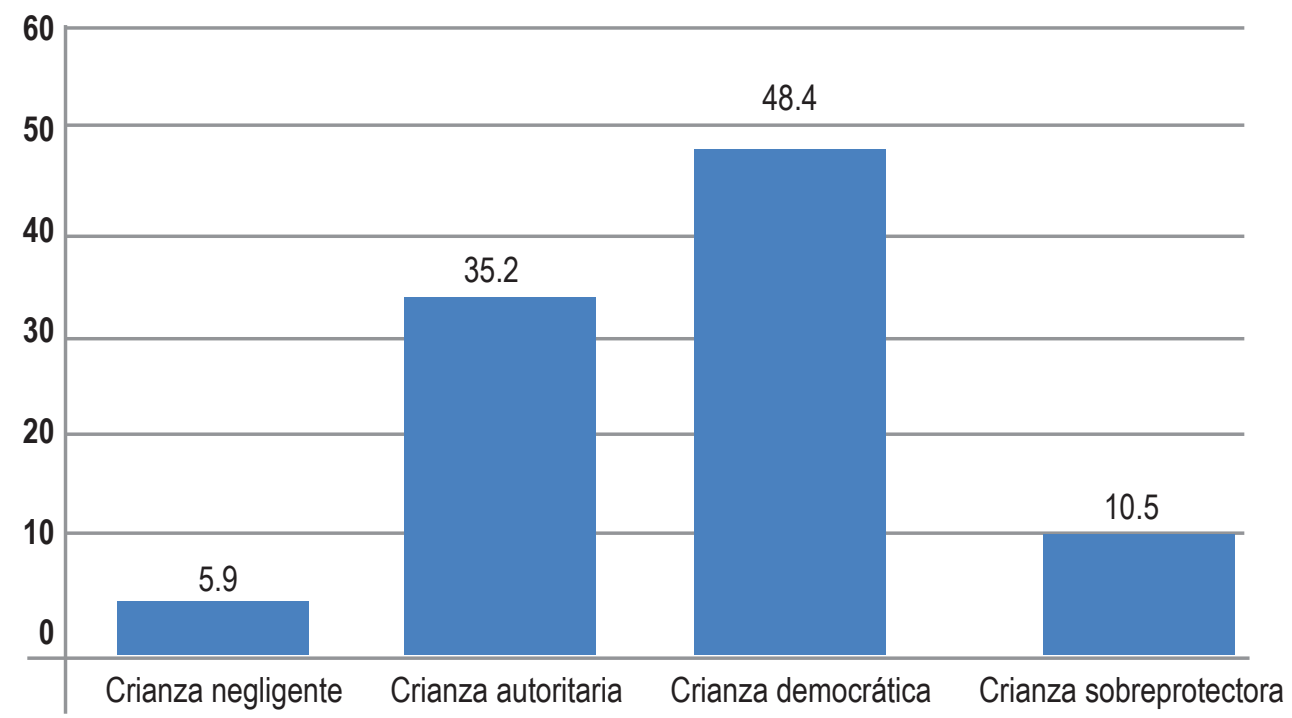

Fuente: elaboración de los investigadores 
Además, es interesante constatar que, pese a las diferencias de tipo étnico que existen en el país, no hay, análogamente, ningún rasgo identitario en ninguna de estas etnias, en relación con las concepciones y a la conciencia de la relación crianza-educación. No hay elementos que nos permitan concluir que hay una relación entre creencias, cosmovisiones y pautas y estilos de crianza.

Se identificó que el tema de la migración está incidiendo en la enseñanza de patrones de crianza; en este sentido, muchos abuelos se han visto obligados a jugar el papel de criadores.

Se pudo también inferir que, detrás de muchas situaciones de conducta negativa o de fracaso escolar de parte de las niñas y niños, se presentan elementos de la atmósfera familiar inadecuados, como el estilo parental autoritario, en que se imponen frente al hijo con una excesiva exigencia.

\section{CONCLUSIONES}

Este estudio, logró describir patrones, pautas y estilos de crianza según la clasificación contextual de las familias hondureñas, además de caracterizar los patrones de crianza en los diferentes grupos étnicos del país. Se pudo establecer, a partir de los roles de género de los jefes (as) de familia, la estructura de prioridades relativas a la crianza y sus implicaciones afectivas, comunicativas y de simetría en las relaciones de los niños y niñas. Fue posible correlacionar las valoraciones de las pautas de crianza empleadas en la actualidad y las pautas de crianza recibidas según los padres de familia a partir de una muestra representativa nacional. Por último, se estimó el tiempo horario dedicado a la crianza según a los roles de género, creencias, profesiones, nivel de escolaridad y contexto urbano rural presente en la muestra representativa nacional de jefes (as) de familia.

En ese sentido, es posible concluir que en Honduras no se han hecho los ajustes necesarios que conlleven a establecer políticas de educación no autoritarias y que los hondureños están bastante influenciados por un tipo de crianza paternalista autoritaria, lo que se explica por el contexto cultural autoritario que aún existe en el país.

Persiste la creencia de que criar consiste solamente en prever las necesidades materiales básicas y que, por lo tanto, "es caro" criar a los hijos, porque "los hijos cuestan". Además de esto, en Honduras hay una tendencia muy marcada a aceptar y mantener 
los roles tradicionales entre hombres y mujeres. Los datos sugieren que, al menos en teoría, persiste la creencia de que los hombres son los que deben proveer lo materialmente necesario para la crianza, mientras que la mujer debe quedarse en su casa cuidando a los hijos.

El estudio permite concluir que, en Honduras, los padres que practican el estilo de crianza democrático, procuran realizar acciones que promuevan la actividad lúdica en los niños y la posibilidad de identificar las preferencias de los menores en esta área. Contrario a los padres que practican el estilo de crianza negligente y democrática. Aunado este factor, los espacios de recreación en las ciudades hondureñas, no permiten el desenvolvimiento y los espacios de esparcimiento. Hoy, a penas se están construyendo centros recreativos en barrios y colonias, los cuales resultan insuficientes, ante el evidente crecimiento poblacional de las grandes ciudades.

El estudio nos permite aseverar que el aspecto económico influye, indudablemente, de alguna manera en la percepción de los padres sobre sus capacidades de crianza. La presencia de estrés por motivos financieros, o la sensación de tener dificultades financieras, parecen tener efectos negativos en las habilidades para la crianza de los hijos 0 , al menos, parece afectar negativamente en la percepción respecto a esas habilidades de crianza. Al indagar sobre diversos aspectos económicos en los procesos de crianza, la mayoría de las personas encuestadas de bajos ingresos indicaron estar siempre preocupados por el dinero para la crianza de sus hijos(as), a diferencia de aquellos con altos ingresos, quienes indicaron nunca sentir preocupación en ese aspecto.

Una conclusión muy importante del estudio es que hay una estrecha relación entre: nivel económico, tiempo dedicado a la crianza y estilo de crianza. Entre menos recursos económicos posee la familia, menos tiempo se le dedica al cuidado de sus hijos, menos satisfacción hay en la crianza, menos tolerantes y más autoritarios, se vuelven los padres.

Así, para los hondureños, las preocupaciones financieras cumplen un rol en esas diferencias tan marcadas. El tema del dinero representa, entonces, una de las prioridades en la crianza de los hijos pues, independientemente del rol que las personas encuestadas ejercen en sus familias, la mayoría coincide en que la mayor responsabilidad de un padre es dar seguridad económica a sus hijos(as); aquí prevalece la idea de que el rol de los padres tanto en las familias como en los procesos de crianza es, en primer lugar, la de ser proveedor para sus hijos e hijas. 
Otro factor asociado a la crianza, es la religión. A grandes rasgos, al indagar sobre los hábitos de crianza entre las familias encuestadas, las respuestas obtenidas por padres y madres católicas fueron en su mayoría, similares a las respuestas brindadas por las familias evangélicas. ¿Será acaso que estas dos denominaciones, son menos dogmáticas, menos basadas en la ley, menos fundamentalistas? De igual manera, los padres y madres de religión mormona siguen una tendencia muy similar, pero con algunas variantes, a pesar de que las diferencias en los principios religiosos entre la iglesia mormona y la católica o evangélica son más pronunciadas. Por su parte, las familias adventistas y testigos de Jehová fueron las que presentaron más diferencias en comparación con las respuestas dadas por las familias de las otras religiones mencionadas anteriormente. La mayoría de personas encuestadas, entrevistadas y que participaron en grupos colaborativos, fueron mujeres; sin embargo, en el caso de las familias mormonas, la mayoría de participantes en la encuesta fueron hombres, a diferencia de las evangélicas y las adventistas en el que todas las personas encuestadas fueron mujeres, pues ningún hombre participó en el cuestionario.

Aunque todas las denominaciones cristianas tienen una enorme preocupación por la crianza de sus hijos, quizás la intensidad y las exigencias de algunas de ellas sean más pronunciadas. Sería quizás el caso de la denominación adventista, cuyo enfoque "se centra en el crecimiento espiritual. Pero también enfatiza elecciones saludables de estilo de vida y aprendizaje intelectual que lleven al servicio útil en la comunidad y el mundo". En algún grado esas exigencias, quizás algo más dogmáticas, hacen que los padres vean difícil la crianza de sus hijos y que no encuentren algún punto de satisfacción en su rol de criadores.

Otro factor que influye en la crianza de los hijos en Honduras, es el número de hijos. En efecto, a mayor número de hijos, más la crianza se hace difícil y más los criadores caen en el peligro de ser negligentes y autoritarios. Paralelamente, los criadores sobreprotectores, no permiten el cultivo de competencias sociales, no permitiendo que los niños controlen su agresividad y sus impulsos, haciendo de ellos niños inmaduros y pocos motivados.

Hay también, una relación muy estrecha entre grado de escolaridad y número de hijos: en Honduras, entre menos es el grado de escolaridad, más se procrean hijos y, viceversa.

Al comparar los dos datos que venimos de enunciar, podemos interpretar que no ha habido ningún progreso notorio en el intervalo de dos generaciones, entre la relación: acceso a la educación, crianza y número de hijos; si las abuelas y abuelos, sin ningún 
nivel de escolaridad, manifiestan haber tenido entre cuatro y cinco hijos; $y$ las mujeres y hombres de la generación siguiente, que dicen haber accedido a la educación básica y secundaria, también afirman tener entre 4 a 5 hijos, se hace necesario preguntarse: ¿Qué papel está jugando la educación en la formación de padres responsables frente a la sexualidad y a la reproducción? Además, es claro que los patrones de crianza destinados a la educación sexual y a los valores que definen a la familia responsable no han evolucionado de una generación a otra, o que pone en tela de juicio el papel responsable que juegan los criadores de una generación a otra. Por otro lado, se ve que, en Honduras, el número de hijos incide severamente sobre las percepciones de los padres y madres, a cerca de su rol de ser criadores: entre más hijos se tiene, más se confunde el sentimiento de ser padre. Podemos deducir por ahí que, frente a estos sentimientos, los padres y madres se vuelven autoritarios y se quejan de que este papel es muy difícil de asumir. Recordemos que la tendencia es que el estilo de crianza es de tipo democrático, seguido muy de cerca (13 puntos) del estilo autoritario.

Además, en Honduras, el número de hijos afecta también la percepción frente a la aceptación de sus hijos, debido a las dificultades económicas, a la poca escolaridad y las pocas posibilidades de desarrollo personal y social, tanto de los padres, como de los hijos.

Así, la satisfacción de ser padre o madre y de tener hijos, varía de acuerdo con el número de hijos: entre más hijos, menos satisfacción de ser padre y, viceversa.

Vemos claramente la relación: rol de padre/madre, cuidado de los hijos, trabajo profesional y número de hijos. Parece que, en Honduras, los padres, cuyas familias están conformadas con un número grande de hijos, piensan que la madre no debería trabajar, sino que debería dedicarse al cuidado de sus hijos y que los padres deben ser los proveedores económicos.

Los resultados que acabamos de mostrar nos revelan que en Honduras la crianza de hijos está siendo afectada por variables como el ingreso económico, el número de hijos y el nivel de escolaridad de os criadores. Se evidencia que, entre los factores que relacionan directamente a los padres y las madres hondureñas con los estilos educativos, el más sobresaliente es el del nivel socioeconómico; aunque en nuestro estudio este factor no refleja exactamente la complejidad de la estructura social, ya que se basa en comparación de grupos relativamente extremos. Parece que dentro de esa variable de nivel socioeconómico (nivel educativo, profesión nivel de ingresos, etc.), el nivel de escolaridad es el que nos ayuda a diferenciar a unos padres de otros 
en los estilos de crianza. De ahí el hecho de que, a mayor escolaridad, menor número de hijos y, viceversa.

La interpretación de los datos arrojados por los diferentes componentes metodológicos, nos permiten interpretar que hay una fuerte influencia de la estructura familiar, sobre las características de las prácticas de crianza; por ejemplo, en las familias extendidas, parece haber menos dificultad para la crianza y para impartir la disciplina. Podemos también afirmar que, en Honduras, la relación entre: la percepción de la madre, la conducta en las prácticas de crianza y la estructura familiar es bastante significativa con el nivel educativo de las madres, aunque también se relaciona con el número de niños en la familia y con los recursos económicos.

Nuestro estudio refleja que un porcentaje significativo de personas participantes forma parte de una familia extensa. En contraposición, un porcentaje similar, dijo provenir de una familia nuclear; este último concepto, es cada día más difícil de abordar, debido a la fuerte tendencia a los divorcios, al auge de madres solteras, etc.

Un hallazgo muy importante es que, a los hondureños, no se les ha enseñado a asimilar el término crianza, no se les ha enseñado a conceptualizarlo debidamente, a que lo hagan parte de un universo simbólico significativo; el término se ha quedado relegado y relacionado a una actividad muy práctica, diaria, "inconsciente", consistente únicamente en proveer alimento, techo y vestido a los hijos. La responsabilidad de los padres parece resumirse y terminar ahí. Temas como la sexualidad, los valores, la moralidad, son muy pocos o nunca abordados.

El hecho de que nuestro estudio revele, además, que uno de los patrones de crianza más fuerte para la mayoría de los hondureños, provenga de doctrinas religiosas, a veces muy dogmáticas, quizás poco pragmáticas, nos deja al desnudo la necesidad de establecer políticas nacionales públicas claras respecto a los contenidos racionales y prácticos que deben formar parte de una crianza, para, a mediano y largo plazo, reducir y acabar con muchos de los males que acongojan a la sociedad hondureña.

\section{RECOMENDACIONES}

Se recomienda el establecimiento de una política pública nacional, respecto a los contenidos racionales (orientación en programas de pautas y estilos de crianza), educativos (generación de un plan de educación, con objetivos específicos y metodo- 
logías específicas) y prácticos, que deben formar parte de la crianza de las niñas y niños hondureños. Así, a mediano y a largo plazo, se podrá reducir y acabar con muchos de los males que acongojan a la sociedad hondureña.

Dicha política nacional pública, debe incluir programas que ayuden a los padres a hacer frente a los problemas de orden económico, con la creación de fuentes dignas de trabajo; debe, además, trabajar en propuestas de prevención en salud mental para las madres y padres, enfrentados al estrés y a la depresión, ya que estas conductas necesariamente inciden en el desarrollo social y emocional de los hijos.

\section{AGRADECIMIENTOS}

Extendemos nuestros más sinceros agradecimientos a la Dirección de Investigación Científica y Posgrado de la UNAH, por su apoyo sostenido en la realización de esta investigación; a los jóvenes alumnos, aprendices investigadores de las carrera de filosofía y de pedagogía, quienes participaron en el proceso. A las personas que participaron de las comunidades de San Juancito, F.M.; El Carrizalón, Copán; Sambo Creek, La Ceiba; la Esperanza, Intibucá; el Progreso Yoro. A todas las personas que apoyaron de manera directa e indirecta en la realización de este estudio.

\section{BIBLIOGRAFÍA}

Bourdieu, Pierre, (1972), Esquisse d'une theorie de la pratique. Genève, Paris: Ed. Droz

Cabrera, V., Guevara, I. \& Barrera, F. (2006). Relaciones maritales, relaciones paternas y su influencia en el ajuste psicológico de los hijos. Acta Colombiana de Psicología, 9 (2), 115-126

Casas F. (1998), Infancia: Perspectivas psicosociales. Barcelona: Paidós.

Chávez, M. \& González, I. (2008). Presencia de la depresión perinatal y la relación madre infante. Estrategia metodológica para una mirada sociocultural. Diversitas, 4 (1), 101-111.

Kant (1985). Tratado de pedagogía, trad. Maldonado, C.E. Bogotá: Ediciones Rosaristas.

Piaget, J. (1971). Epistemología y Psicología de la Identidad (10 Ed.) Buenos Aires: Paidós 
Piaget, J. (1980). Adaptación Vital y Psicología de la Inteligencia. ( $3^{\circ}$ Ed.) Madrid: Siglo Veintiuno Editores. Piaget, J. (1991). Seis Estudios de Psicología. (1 Ed.). Barcelona: Editorial Labor.

Reboul, Olivier (2012), la philosophie de l'éducation, col. que sais je ?. puf, Paris Rich D. (1998), Hijos, ¿Qué hacemos? ¿Qué les decimos? Buenos Aires: Vergara,

Rodriguez, Gomez, GilFlores, Garcia Jimenez, (1996). Metodología de la investigación cualitativa, Granada: Ediciones Aljibe

Sánchez Gamboa (1998). Fundamentos para la investigación educativa: presupuestos epistemológicos que orientan al investigador. Colombia: Editorial Mesa Redonda, Magisterio, 1998, pág., 61.

Savater, Fernando (1997). El valor de educar, Barcelona: ed. Ariel.

Schaefer, E. S. (1959) A circumplex model for maternal behavior. Journal of Abnormal Social Psychology 59, 226-235

Searle, Jonh R. (2012). La construcción de la realidad social. Barcelona: Paidós 